\title{
Desenvolvimento motor de crianças entre o e 18 meses de idade: Diferenças entre os sexos
}

\author{
Motor development of children between o and 18 months of age: \\ Differences between sexes
}

\author{
C.B. Venturella, G. Zanandrea, R. Saccani, N.C. Valentini
}

ARTIGO ORIGINAL | ORIGINAL ARTICLE

\begin{abstract}
RESUMO
Os primeiros anos na vida da criança são fundamentais para a aquisição de habilidades motoras, cognitivas e sociais; desta forma as oportunidades ofertadas as crianças e as expectativas sociais podem interferir no desenvolvimento. O objetivo deste estudo foi avaliar e comparar o desenvolvimento motor de meninos e meninas na primeira infância. Participaram deste estudo, 90 crianças de 0 a 18 meses (45 meninas e 45 meninos pareados por idade), residentes no sul do Brasil, provenientes de Escolas de Educação Infantil. A Alberta Infant Motor Scale (AIMS) foi utilizada para avaliar o desempenho motor. A maioria dos participantes $(73.3 \%)$ apresentou desempenho motor normal; não foram observadas diferenças significativas no desenvolvimento motor entre os sexos nos escores da AIMS ( $p_{\text {esc.total }}=.76$; $\left.p_{\text {percentil }}=.38\right)$ e nas diferentes posturas $\left(p_{\text {prono }}=.71 ; p_{\text {supino }}=.49 ; p_{\text {sentado }}=.71 ; p_{\text {empé }}=.97 ;\right.$, não foram observadas associações entre sexo e desempenho motor $\left(\operatorname{Eta}^{2}=.008\right.$; $\left.\operatorname{Eta}^{2}=.108\right)$. Nos primeiros dois anos de vida, meninos e meninas demonstram desempenho motor amplo similar. Infere-se que as diferenças entre sexo que aparecem com o passar do tempo são influenciadas pelas oportunidades do contexto.
\end{abstract}

Palavras-chave: desenvolvimento infantil, destreza motora, fatores de risco

ABSTRACT

The first years of life are fundamental to the acquisition of motor, cognitive, and social skills; therefore the opportunities provide to the child as well as the social' exceptive affect the development. The objective of this study was to assess and compare the motor development of boys and girls ages from 0 to 18 months. Ninety infants, ages from 0 to 18 (45 boys and 45 girls, stratified by age groups), that lived in the south of Brazil, attending to daycares participated in the present study. The Alberta Infant Motor Scale (AIMS) was used to evaluate motor development. The majority of the infants showed typical development (73.3\%); no significant differences between the sexes was found for motor development for the AIMS total scores $\left(p_{\text {esc.total }}=.76 ; p_{\text {percentil }}=.38\right)$ and postures $\left(p_{\text {prono }}=.71 ; p\right.$ supino $=.49 ; p_{\text {sentado }}=$ $.71 ;$ empé $=.97 ;$ pesc.total $=.76 ;$ percentil $=.38)$; non-significant differences' for association between motor development and sex were found $\left(\mathrm{Eta}^{2}=.008 ; \mathrm{Eta}^{2}=.108\right)$. In the two first years of life, boys and girls demonstrated similar motor development performance. It is conjectured that the differences between sexes observed latter is affected by opportunities of the environment.

Keywords: child development, motor skills, risk factors

Submetido: 09.05.2012 | Aceite: 08.04.2013

Cíntia Barreto Venturella, Gabriela Zanandrea, Raquel Saccani. Universidade de Caxias do Sul - UCS, Brasil.

Nadia Cristina Valentini. Universidade Federal do Rio Grande - UFRGS, Brasil.

Endereço para correspondência: Raquel Saccani, Avenida Loureiro da Silva, 1788, apt 209, CEP 90050240, Porto Alegre, RS, Brasil.

E-mail: raquelsaccani@yahoo.com.br 
Os possíveis fatores de risco que influenciam nas aquisições comportamentais da criança têm sido alvo de diversas pesquisas (Eikmann et al., 2007; Halpern, Giuliani, Victora, Barros, \& Horta, 2000; Pierce, Munier, \& Myers, 2009; Pretti, Milan, \& Foschiani, 2010; To et al., 2004). Dentre estes, se destacam, os fatores relacionados à especificidade das tarefas ofertadas à criança (Haydari, Askari, \& Nezhad, 2009; Nobre et al., 2009), assim como os fatores socioeconômicos e ambientais, os quais potencializam atrasos no crescimento e desenvolvimento infantil (Eikmann et al., 2007; Hamadani et al., 2010).

Considerando os tipos de estímulos ofertados as crianças, pesquisadores demonstram esforços para identificar as consequências do tipo de tarefas e atividades as quais a criança é exposta (Pin, Eldridge, \& Galea, 2007). Frente a isso, as diferenças no desempenho e habilidades motoras entre meninos e meninas se tornaram alvo de preocupações, pois a indução de atividades específicas para cada sexo pode influenciar as aquisições motoras das crianças. Essas distinções, na maioria das vezes são determinadas pelo contexto e pelas atividades que são propostas a criança, através de brinquedos e atividades impostas pelo padrão cultural considerado mais apropriado para cada um deles (Schwengber, 2009).

Teóricos do desenvolvimento humano ressaltam que as diferenças entre os sexos estão relacionadas às expectativas pessoais e sociais vivenciadas pela criança desde o seu nascimento, as quais podem direcionar seus comportamentos (Papalia, Olds, \& Feldman, 2010; Haywood \& Getchell, 2010). Esta influência na formação do indivíduo se refere a três aspectos fundamentais: papeis de gênero (atitudes, habilidades e traços de personalidade considerados apropriados para meninos e meninas segundo contexto sócio cultural); tipificação de gênero (crianças passam a aprender características consideradas apropriadas a cada sexo); e estereótipos de gênero (generalizações predeter- minadas sobre o comportamento adequado ao masculino e feminino) (Papalia et al., 2010). Sendo assim, a abordagem desenvolvimentista afirma que todos os seres humanos são seres sociais, ou seja, desde o período pré-natal se desenvolvem dentro de um contexto histórico, social e cultural preestabelecido (Papalia et al., 2010; Gallahue \& Ozmun, 2005; Haywood \& Getchell, 2010).

Assim, as crianças aprendem os papeis relacionados aos gêneros, masculino e feminino, por meio da socialização (Papalia et al., 2010). Diante disto, não há dúvidas de que os indivíduos apresentam diferenças sexuais determinadas biologicamente, no entanto, as características de cada um não estão relacionadas apenas ao sexo, mas sim aos valores familiares, bem como a fatores sociais, econômicos e culturais. Consequentemente, as disparidades no comportamento motor entre os sexos surgem com o avançar da idade (Gallahue \& Ozmum, 2005).

Estudos recentes com crianças acima dos 4 anos demonstram que fatores ambientais influenciam nas aptidões de cada criança (Cardoso, 2008; Schwengber, 2009). Outras pesquisas com crianças em idade escolar, constaram diferenças de desempenho entre meninos e meninas em diversos testes motores analisados e associam essa discrepância, principalmente, às oportunidades propiciadas para cada sexo (Valentini, 2002; Carvalhal \& Vasconcelos-Raposo, 2007). Porém, estudos na primeira infância sugerem que meninos e meninas apresentam desenvolvimento motor semelhante durante os primeiros 2 anos de vida (Saccani \& Valentini, 2010; Eikmann et al., 2007).

É fundamental diagnosticar, precocemente, se o desenvolvimento esta ocorrendo de forma típica ou se nos primeiros anos de vida, já está sendo influenciado pelo contexto e pelas tarefas ofertadas às crianças. Uma das preocupações, portanto, é detectar em que período do desenvolvimento as diferenças na trajetória são observadas, para uma posterior investigação das causas destas diferenças. O estudo 
das diferenças entre meninos e meninas tem sido conduzido por décadas (Thomas \& French, 1985). Embora, considerando o desenvolvimento motor, se observa maior incidência de estudos direcionados a faixas etárias acima dos 4 anos de idade (Cardoso, 2008; Miranda, Resegue, \& Figueiras, 2003; Schwengber, 2009) e poucos estudos em crianças com menos de 2 anos enfocando desenvolvimento motor (Eikmann et al., 2007; To et al., 2004; Lung et al., 2011). Ainda mais, os resultados, em geral, são parciais e contraditórios quanto as diferenças motoras nos primeiros 2 anos de vida. Além disso, muitos estudos não contemplam todas as faixas etárias (Lung et al., 2011; WHO Multicenter Growth Reference Study \& Onis, 2006) ou não consideram aspectos motores (Eikmann et al., 2007; Gabbard, Caçola, \& Rodrigues, 2009), embora indiretamente, indicam semelhanças motoras entre os sexos até os dois anos de idade (Eikmann et al., 2007; Saccani \& Valentini, 2010).

Considerando que a literatura assegura que as brincadeiras e tarefas oferecidas para meninos e meninas predispõem a um crescimento e desenvolvimento diferenciado entre eles (Wanderlind, Martins, Hansen, Macarini, \& Vieira, 2006) a investigação das diferenças motoras entre sexos se torna essencial mesmo antes dos dois anos; porém, em delineamentos que contemplem as diferentes idades em meses (exemplos: transversal e longitudinal) uma vez que as mudanças no desenvolvimento motor são observadas cotidianamente (Clark \& Metcalfe, 2002). Sendo assim, esta pesquisa se propôs a avançar o conhecimento atual na área, investigando as diferenças entre os sexos no que se refere ao desenvolvimento motor de meninos e meninas, do nascimento até o caminhar independente. Foi estabelecida como hipótese a não observação de diferenças significativas entre os sexos no desenvolvimento motor amplo nas faixas etárias investigadas.

\section{MÉTODO}

\section{Amostra}

Estudo descritivo e observacional de caráter associativo e comparativo com delineamento transversal, onde foi observado o desempenho dos bebês em um único momento avaliativo e feita associação entre variáveis qualitativas e correlação entre variáveis quantitativas (Thomas \& Nelson, 2007). Foi aprovado pelo Comitê de Ética em Pesquisa da Universidade Federal do Rio Grande do Sul ( ${ }^{\circ}$ 2002018).

Os participantes foram crianças $(\mathrm{N}=90)$ de 0 a 18 meses de idade, de ambos os sexos, provenientes de Escolas de Educação Infantil Particulares (EEIP's) do Município de Caxias do Sul, no sul do Brasil. Foram excluídas crianças com: 1) Baixo peso ao nascer (< $1500 \mathrm{~g})$; 2) Prematuridade; 3) Alterações neurológicas; 4) Problemas osteomioarticulares (fraturas; lesões nervosas periféricas, infecção osteomuscular, entre outras citadas pelos cuidadores); 5) Idade materna precoce e avançada. Além disso, foram excluídos do estudo crianças participantes de programas de intervenção. Os grupos, masculino (GM, $\mathrm{N}=45)$ e feminino ( $\mathrm{GF}, \mathrm{N}=45$ ) foram pareados por idade. A renda familiar dos participantes variou entre 1 e 5 ou mais salários mínimos, sendo que a maior porcentagem da amostra (50\%) apresentava 5 ou mais salários mínimos (GM: 57.8\%; GF:42.2\%). A escolaridade dos pais também foi considerada, onde se observou maior porcentagem de pais com ensino superior (38.9) e de mães com ensino médio completo (42.2\%).

\section{Instrumentos}

Para avaliar o desenvolvimento motor foi utilizada a Alberta Infant Motor Scale (AIMS) com a versão adaptada e validada para crianças brasileiras (Valentini \& Saccani, 2011). A AIMS é uma escala de avaliação observacional, empregada para medir a evolução das aquisições motoras amplas em bebês, a partir de seu nascimento até o alcance da marcha independente (0 a 18 meses de idade), através de manuseios 
mínimos, com duração média de vinte minutos.

A AIMS enfatiza a sequência do desenvolvimento do controle postural quando em quatro posturas básicas: prono, supino, sentado e em pé. É composta por 58 itens, divididos nas quatro posições posturais das crianças, 21 posturas em prono; 9 em supino; 12 sentado e 16 na posição em pé, avaliando em cada item três aspectos do comportamento motor: postura, movimentação antigravitacional e a superfície corporal em que ocorre a sustentação do peso. Durante a avaliação deve ser observado o desempenho motor da criança nas posturas, atribuindo 1 ponto para cada critério motor observado e 0 ponto para cada critério não observado. A escala disponibiliza investigar o desempenho da criança por meio de escore bruto, percentil e categorização do desempenho. O escore total bruto varia de 0 a 58 pontos e resulta da soma de itens nas 4 posturas. A curva percentílica é obtida a partir da relação do valor bruto com a idade da criança. Além disso, os percentis permitem inferir o status do desempenho motor do bebê, categorizando os resultados de acordo com os seguintes critérios: a) desempenho motor normal/esperado quando o resultado no teste estiver acima de $25 \%$ na curva percentílica; b) desempenho motor suspeito quando a o resultado estiver entre $25 \%$ e $6 \%$ na curva percentilica; c) desempenho motor anormal quando o resultado for menor ou igual a $5 \%$ na curva poercentilica (Piper, Pinnell, Darrah, Maguire, \& Byrne, 1992)

O questionário referente às condições biológicas e sócio-ambientais do bebê pesquisou os seguintes aspectos: data de nascimento; semanas de gestação; índice de APGAR no $1^{\circ}$ e $5^{\circ}$ minuto; idade materna; peso ao nascer; comprimento ao nascer; renda familiar e escolaridade dos pais.

\section{Procedimentos}

Inicialmente, foram apresentados os objetivos e as propostas deste estudo aos dire- tores e/ou coordenadores das escolas. Após o consentimento das escolas, os pais ou responsáveis legais das crianças foram contatados quanto ao interesse de seus filhos participarem do estudo. As crianças foram avaliadas somente após a assinatura do Termo de Consentimento Livre e Esclarecido (TCLE).

Foram agendados horários com as EEIP's para realizar as avaliações, e enviado os questionários aos pais sobre informações socioeconômicas. Durante as avaliações, conduzidas por dois avaliadores independentes, treinados no uso da AIMS, os bebês se encontravam vestidos com roupas confortáveis que garantiam a livre movimentação. As avaliações ocorreram nas dependências das instituições, sobre colchonetes ou dispositivos fornecidos pelo local. Todas as avaliações foram registradas em filmagem (câmera SONY w80), as quais foram usadas unicamente para fins científicos, sem exposição dos participantes, os quais tiveram sua identidade preservada. As crianças foram avaliadas por meio da observação dos 58 itens da AIMS distribuídos nas quatro posturas, com o mínimo de manuseios e facilitações. Durante a avaliação, o examinador observou a movimentação da criança em cada uma das 4 posições, considerando nesta observação, como a criança apoia a superfície do corpo, ou seja, como sustenta seu peso, além da qualidade da postura e dos movimentos antigravitacionais. Foram oferecidos estímulos visuais, auditivos e verbais para encorajar a criança a adquirir as posturas desejadas, não sendo indicado a manipulação da criança. Após avaliar os itens pertencentes aquelas janelas do estágio de desenvolvimento motor, o examinador somou os pontos creditados nas quatro posturas para obter o escore total da AIMS.

Após coleta de dados, as filmagens foram utilizadas para análise da objetividade entre os dois avaliadores. Os coeficientes de correlação oscilaram entre $\alpha=.86$ e $\alpha=.99$. Estes resultados são considerados adequados e estão de acordo com o proposto pelas autoras do teste 
que sugerem adequados os valores acima de 80\% (Piper et al., 1992).

\section{Análise Estatística}

Os dados dessa pesquisa foram organizados e analisados no programa estatístico SPSS 17.0 (Statistical Package to Social Sciences for Windows). Foi utilizada estatística descritiva (frequência simples e relativa, medidas de tendência central e de variabilidade) para descrever o desenvolvimento motor dos bebês participantes. Para as associações e correlações do desempenho foi utilizado o Teste qui-quadrado de Pearson $\left(\mathrm{Chi}^{2}\right)$, teste $\mathrm{Eta}^{2}$ e Correlação de Pearson. Como critério de decisão, para o $\mathrm{Chi}^{2}$, foi considerado $p \leq .05$ e para o Eta $^{2}$ e Correlação de Pearson foi considerado: associações/correlações fracas valores abaixo e iguais a 0.30; associações/correlações moderadas incluíram valores entre 0.30 e 0.60 ; e associações/correlações fortes quando os valores incidissem acima de 0.60. A distribuição dos dados foi investigada, inicialmente, com o teste Shapiro-Wilk. Teste $t$ student foi utilizado na comparacão dos grupos e o teste U Mann-Whitney na comparação entre os sexos, por trimestres, devida amostra reduzida. Como critério de decisão, o nível de significância adotado foi $p \leq .05$ (Callegari-Jaques, 2003).

\section{RESULTADOS}

\section{Descrição da amostra}

A amostra do presente estudo foi composta por 90 crianças, sendo 45 (50\%) do sexo masculino e 45 (50\%) do sexo feminino, cuja média de idade, foi de 10.36 ( \pm 4.70$)$, entre 0 a 18 meses. Nos agru- pamentos por faixa etária (trimestres), a amostra se distribuiu nas seguintes frequências: 6 bebês (6.7\%) no primeiro trimestre (0 a 3 meses), 16 bebês (17.8\%) no segundo (4 a 6 meses), 16 bebês (17.8\%) no terceiro trimestre (7 a 9 meses), 16 bebês (17.8\%) no quarto (10 a 12 meses), 20 bebês (22.2\%) no quinto trimestre (13 a 15 meses) e 16 bebês (17.8\%) no sexto (16 a 18 meses); equivalentemente distribuídos nos grupos (GM e GF).

Com relação às características biológicas, não foram encontradas diferenças significativas entre os sexos, conforme demonstrado na Tabela 1, assim como se observa padrão de normalidade para cada uma das variáveis analisadas.

\section{Comparação do desempenho motor de meni- nos e meninas}

Os dados referentes ao desenvolvimento motor geral das crianças, nas diferentes posturas analisadas da AIMS, estão dispostos na Tabela 2, assim como o escore total e percentil da amostra estudada. Ao comparar o desenvolvimento motor entre os sexos, não foram observadas diferenças significativas $(p \leq$ .05) nas posturas analisadas, nem no escore total e no percentil $\left(p_{\text {prono }}=.71 ; p_{\text {supino }}=.49 ; p_{\text {sentado }}=.71\right.$; $\left.p_{\text {empé }}=.97 ; p_{\text {esc.total }}=.76 ; p_{\text {percentil }}=.38\right)$.

A análise do desenvolvimento com base no critério de categorização de desempenho motor, está disposta do Gráfico 1, onde se observa que grande número das crianças analisadas apresentou desenvolvimento motor normal (73.30\%). No grupo GM, nenhum caso de atraso motor foi observado e apenas 1 criança do GF foi classificada com atraso no desenvolvimento motor. No Gráfico 1, estão dispostos os dados referentes a categorização da amostra de forma geral e segundo sexo.

Tabela 1

Medidas de tendência central e variabilidade das características biológicas dos participantes segundo grupos analisados

\begin{tabular}{cccc}
\hline Características & GM & GF & $p(<.05)$ \\
\hline Idade (meses) M + DP & $10.36 \pm 4.70$ & $10.36 \pm 4.70$ & 1.0 \\
Idade materna (anos) M + DP & $30.24 \pm 5.51$ & $29.86 \pm 5.63$ & .80 \\
Idade gestacional (semanas) M + DP & $38.58 \pm 1.17$ & $38.81 \pm 1.47$ & .50 \\
Comprimento ao nascer (cm) M + DP & $48.72 \pm 2.15$ & $46.62 \pm 9.78$ & .24 \\
Peso ao nascer (g) M + DP & $3243.48 \pm 537.17$ & $3284.26 \pm 450.47$ & .75 \\
Apgar 1' Md (P25-P75) & $9(9-9)$ & $9(8.25-9)$ & .65 \\
Apgar 5' Md (P25-P75) & $9(9-10)$ & $9(9-10)$ & .39 \\
\hline
\end{tabular}


Foi realizada comparação do desempenho motor apresentado por meninos e meninas nas diferentes faixas etárias (tabela 3), embora tenha sido observada correlação fraca do desempenho motor dos participantes com o fator idade $(r=.22 ; p=.04)$. Em cada um dos trimestres analisados, não foram encontradas diferenças significativas em nenhuma das variáveis, como pode ser observado na Tabela 3.

Reafirmando os resultados acima citados, de igualdade entre meninos e meninas, a análise associativa entre o sexo da criança e o percentil da AIMS, não revelou resultados significativos (Eta $=.093$; $\left.\mathrm{Eta}^{2}=.008\right)$, assim como, o tratamento estatístico não detectou associação significativa entre os sexos masculino e feminino, com o escore total AIMS $\left(E t a=.033 ; \mathrm{Eta}^{2}=.108\right)$. Ao considerar o critério de classificação, também não foi detectada associação estatisticamente significativa entre o sexo do bebê e a classificação de desempenho motor $\operatorname{AIMS}\left(\mathrm{Chi}^{2}=\right.$ 1.634; $p=.442$ ), ou seja, esses resultados demonstram que, na faixa etária avaliada, o sexo da criança não influenciou no desempenho motor.

\section{DISCUSSÃO E CONCLUSÕES}

A análise do desempenho dos participantes demonstrou que a maioria (73.30\%) apresentou desenvolvimento motor adequado; congruente com resultados de estudos nacionais utilizando o mesmo instrumento (Manacero \& Nunes, 2008; Zanini et al., 2002). Entretanto, contrapõem outros estudos brasileiros com crianças na mesma faixa etária que demonstraram desempenho motor inferior ao esperado para idade (Formiga \& Linhares, 2011; Halpern et al., 2000; Lopes, Lima, \& Tudella, 2009; Saccani \& Valentini, 2010; Santos et al., 2004).

A hipótese inicial desta pesquisa foi confirmada. Não foram encontradas diferenças significativas entre o desempenho motor de meninos e meninas até os 18 meses em nenhum dos trimestres. A semelhança entre os sexos nas diferentes posturas analisadas estão de acordo com outros estudos prévios que evidenciam poucas diferenças no desenvolvimento motor nessa fase (Eikmann et al., 2007; Fleuren, Smit, Stijnen, \& Hartman, 2007; Lung et al., 2011; Saccani \& Valentini, 2010; WHO Multicenter Growth Reference Study \& Onis, 2006). Cabe

Tabela 2

Variáveis do desempenho motor dos participantes em geral e por grupos

\begin{tabular}{ccccccc}
\hline & Prono & Supino & Sentado & Em pé & Escore total & Percentil \\
& $\mathrm{M}+\mathrm{DP}$ & $\mathrm{M}+\mathrm{DP}$ & $\mathrm{M}+\mathrm{DP}$ & $\mathrm{M}+\mathrm{DP}$ & $\mathrm{M}+\mathrm{DP}$ & $\mathrm{M}+\mathrm{DP}$ \\
\hline Geral & $15.62 \pm 6.75$ & $8.16 \pm 1.81$ & $9.50 \pm 3.69$ & $8.91 \pm 5.55$ & $42.23 \pm 16.43$ & $49.29 \pm 25.40$ \\
$\mathrm{GM}$ & $15.89 \pm 6.48$ & $8.29 \pm 1.76$ & $9.64 \pm 3.65$ & $8.93 \pm 5.44$ & $42.78 \pm 15.70$ & $51.64 \pm 24.46$ \\
$\mathrm{GF}$ & $15.36 \pm 7.07$ & $8.02 \pm 1.87$ & $9.36 \pm 3.77$ & $8.89 \pm 5.71$ & $41.69 \pm 17.29$ & $46.93 \pm 26.36$ \\
Valor $p(\leq .05)$ & .71 & .49 & .71 & .97 & .76 & .38 \\
\hline
\end{tabular}

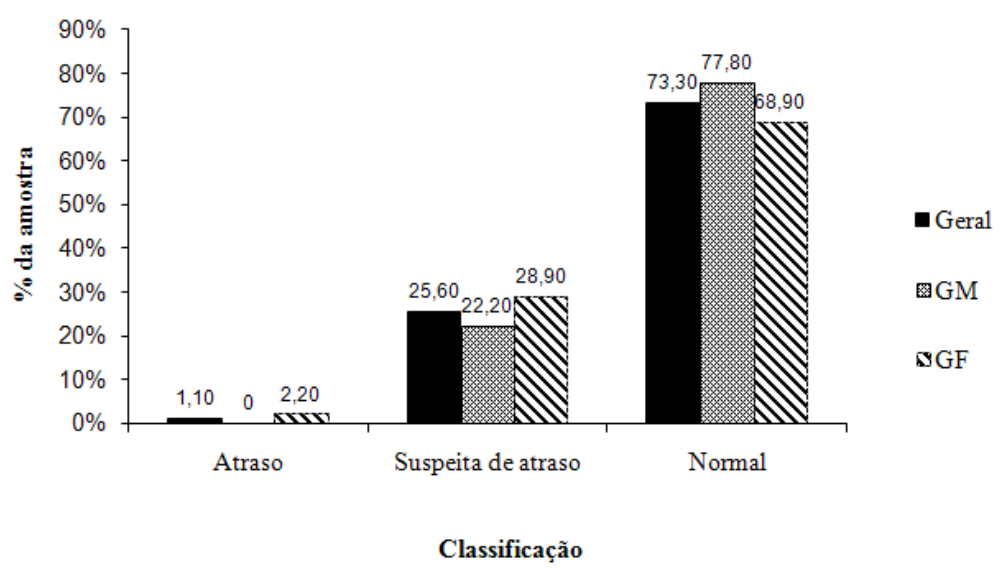

Gráfico 1. Categorização de desempenho motor geral dos participantes e por sexos. 
Tabela 3

Médias e desvios padrão do desempenho motor por posturas, escore total e percentil de cada grupo por faixa etária

\begin{tabular}{|c|c|c|c|c|c|c|c|}
\hline \multicolumn{8}{|c|}{ PONTUAÇÕES AIMS } \\
\hline & & Prono & Supino & Sentado & Em pé & Esc. Total & Percentil \\
\hline TRIMESTRES & Sexo & Média \pm DP & Média $\pm \mathrm{DP}$ & Média \pm DP & Média \pm DP & Média $\pm \mathrm{DP}$ & Média $\pm \mathrm{DP}$ \\
\hline \multirow[t]{2}{*}{$1^{\circ}$ trim. $(n=6)$} & M & $2.67 \pm 2.08$ & $3.00 \pm 1.00$ & $1.00 \pm 0.00$ & $1.67 \pm 0.57$ & $8.67 \pm 3.21$ & $44.00 \pm 17.57$ \\
\hline & $\mathrm{F}$ & $2.67 \pm 2.08$ & $2.33 \pm 0.57$ & $1.67 \pm 1.15$ & $1.67 \pm 1.15$ & $8.33 \pm 4.93$ & $45.67 \pm 34.38$ \\
\hline Valor $p$ & & 1.00 & .35 & .32 & .81 & 1.00 & .66 \\
\hline \multirow[t]{2}{*}{$2^{\circ}$ trim. $(n=16)$} & M & $8.13 \pm 1.80$ & $7.88 \pm 2.10$ & $5.00 \pm 2.26$ & $3.50 \pm 1.19$ & $24.50 \pm 3.96$ & $58.75 \pm 27.66$ \\
\hline & $\mathrm{F}$ & $6.88 \pm 3.39$ & $7.13 \pm 1.45$ & $4.75 \pm 2.60$ & $2.50 \pm 1.06$ & $21.25 \pm 7.00$ & $43.63 \pm 31.05$ \\
\hline Valor $p$ & & .22 & .55 & .96 & .10 & .40 & .29 \\
\hline \multirow[t]{2}{*}{$3^{\circ}$ trim. $(n=16)$} & M & $13.63 \pm 4.40$ & $8.38 \pm 0.91$ & $10.50 \pm 1.51$ & $5.75 \pm 2.05$ & $38.25 \pm 7.96$ & $49.63 \pm 27.52$ \\
\hline & $\mathrm{F}$ & $11.50 \pm 4.95$ & $8.00 \pm 1.30$ & $8.63 \pm 2.82$ & $4.50 \pm 2.20$ & $33.00 \pm 9.59$ & $29.63 \pm 25.82$ \\
\hline Valor $p$ & & .31 & .56 & .13 & .24 & .19 & .13 \\
\hline \multirow[t]{2}{*}{$4^{\circ}$ trim. $(n=16)$} & M & $19.38 \pm 3.11$ & $9.00 \pm 0.00$ & $11.38 \pm 1.18$ & $7.50 \pm 3.25$ & $47.25 \pm 5.72$ & $32.00 \pm 22.04$ \\
\hline & $\mathrm{F}$ & $19.75 \pm 2.31$ & $8.88 \pm 0.35$ & $11.75 \pm 0.46$ & $8.75 \pm 1.90$ & $49.13 \pm 4.79$ & $38.00 \pm 21.88$ \\
\hline Valor $p$ & & .70 & .32 & .78 & .40 & .34 & .49 \\
\hline \multirow[t]{2}{*}{$5^{\circ}$ trim. $(n=20)$} & M & $21.00 \pm 0.00$ & $9.00 \pm 0.00$ & $12.00 \pm 0.00$ & $13.60 \pm 3.20$ & $55.60 \pm 3.20$ & $52.00 \pm 24.34$ \\
\hline & $\mathrm{F}$ & $21.00 \pm 0.00$ & $9.00 \pm 0.00$ & $11.90 \pm 0.31$ & $14.60 \pm 2.27$ & $56.50 \pm 2.41$ & $56.90 \pm 22.83$ \\
\hline Valor $p$ & & 1.00 & 1.00 & .31 & .42 & .47 & .52 \\
\hline \multirow[t]{2}{*}{$6^{\circ}$ trim. $(n=16)$} & M & $21.00 \pm 0.00$ & $9.00 \pm 0.00$ & $12.00 \pm 0.00$ & $15.88 \pm 0.35$ & $57.88 \pm 0.35$ & $68.63 \pm 6.71$ \\
\hline & $\mathrm{F}$ & $21.00 \pm 0.00$ & $9.00 \pm 0.00$ & $12.00 \pm 0.00$ & $15.38 \pm 1.76$ & $57.38 \pm 1.76$ & $64.50 \pm 17.58$ \\
\hline Valor $p$ & & 1.00 & 1.00 & 1.00 & .93 & .93 & .30 \\
\hline
\end{tabular}

ressaltar, que muitos estudos não contemplam, especificamente, diferentes faixas etárias nos dois primeiros anos de vida; e/ou, não utilizaram pareamento amostral. Estudos considerando diferentes faixas etárias e com amostras grandes, por exemplo, Saccani e Valentini (2010), avaliaram 561 crianças brasileiras e Fleuren et al. (2007) na Holanda, através de estudo com 100 crianças de 0 a 12 meses, utilizando o mesmo instrumento, reportaram semelhanças no desempenho de meninos e meninas.

Ainda mais, em um estudo em larga escala, recentemente conduzido em Taiwan, acompanhando 1620 crianças dos 6 aos 60 meses, com avaliações aos $6,18,36$ e 60 meses não observaram associações significativas entre o sexo e o desenvolvimento motor amplo e fino, embora os autores reportem associações positivas e significantes entre os sexos na linguagem, aos 18, 36 e 60 meses, assim como para os aspectos sociais aos 18 e 60 meses e motores finos, aos 36 e 60 meses (Lung et al., 2011). A Organização Mundial da Saúde, desde 2006, demonstra a preocupação com esta linha investigativa, e reporta também semelhança na aquisição de marcos motores de meninos e meninas de diferentes países (Gana, Índia, Noruega e USA) até 24 meses de idade (WHO Multicenter Growth Reference Study \& Onis, 2006).

A influência do contexto e da tarefa tem sido discutida em vários modelos teóricos de desenvolvimento (Clark \& Metcalfe, 2002; Gallahue \& Ozmun, 2005) e comprovada em muitos estudos pesquisando os pais e agentes socializadores que frequentemente induzem as crianças a atividades consideradas adequadas a meninos e meninas, considerando suas características biológicas (Haywood \& Getchell, 2010). Os 
estudos relacionados com as diferenças entre os sexos apresentam resultados de grande variabilidade interindividual, mas com uma tendência dos meninos serem mais hábeis motoramente do que as meninas. Estas diferenças têm sido atribuídas às influências culturais que predominam na sociedade. Meninos são incentivados as vivências motoras mais amplas, vigorosas e expansivas; as meninas as práticas motoras restritas e sedentárias que envolvem predominantemente motricidade fina e que podem limitar seu desempenho motor (Haywood \& Getchell, 2010; Papalia et al., 2010).

Assim, considerando a importância de iguais oportunidades de desenvolvimento, estudos demonstraram que com o avançar da idade, as disparidades no desempenho motor vão se acentuando, se tornando mais expressivas na puberdade (Carvalhal \& Vasconcelos-Raposo, 2007; Valentini, 2002). A diferenciação observada em algumas habilidades ocorre principalmente porque o contexto ambiental e as práticas propostas para cada sexo diferem entre si, de acordo com as expectativas dos pais, dos educadores e do grupo etário do qual as crianças pertencem. Argumenta-se que esta diferenciação é ocasionada pelo padrão sociocultural, onde os fatores educacionais, as perspectivas culturais e as tarefas oportunizadas às crianças levam à distinção de papeis para meninos e meninas (Carvalhal \& Vasconcelos-Raposo, 2007). Portanto, se acredita que na faixa etária estudada ainda não ocorra tanta diferenciação nos tipos de atividades ofertadas, devendo esta, aumentar a partir da aquisição da locomoção independente. Ou ainda, embora diferenciações na qualidade e tipo de estimulação possam ter ocorrido, esta não foi suficiente para gerar diferenças nos desempenhos motores.

As crianças nascem capacitadas para o desenvolvimento de diversas habilidades sensório-motoras, que lhes permitem atingir controle sobre seus corpos, movimentos e emoções (Clark \& Metcalfe, 2002; Haywood \& Getchell, 2010). Contudo o resultado deste controle dependerá não apenas das características do indivíduo, mas também da qualidade de sua interação com o ambiente e com as experiências e tarefas que são oferecidas nestes contextos (Clark \& Metcalfe, 2002; Gabbard et al., 2008).

Considerando os resultados do presente estudo e os achados contraditórios da literatura na mesma faixa etária investigada, se reforça a importância de estudos voltados à identificação precoce de possíveis diferenças entre os sexos, em outras regiões do Brasil, bem como em diferentes classes sociais. Ainda mais, considerar os fatores socioeconômicos e as práticas parentais, assim como sua possível associação com o desenvolvimento motor se faz essência para provar o cuidado adequado na primeira infância. Portanto, se ressalta a importância da inserção de profissionais especializados nessa área de intervenção e diagnóstico precoce das trajetórias de desenvolvimento.

Fica como sugestão a criação e a implantação de programas sociais que atendam as essas questões, assim como, se percebe a necessidade de investimentos em infraestrutura e capacitação pedagógica qualificada, que viabilizem o diagnóstico de um maior número de crianças e o encaminhamento de crianças para avaliações complementares quando detectado o risco ao desenvolvimento. Treinamento de profissionais e programas educativos para os familiares, bem como, das educadoras de escolas infantis é imprescindível para o sucesso da identificação do risco e para as ações concomitantemente às necessidades de desenvolvimento do bebê.

\section{Agradecimentos:}

Nada declarado.

\section{Conflito de Interesses:}

Nada declarado.

Financiamento:

Nada declarado. 


\section{REFERENNCIAS}

Callegari-Jaques, S. M. (2003). Bioestatística: Princípios e aplicações. Porto Alegre: Artmed.

Cardoso, F. L. (2008). O conceito de orientação sexual na encruzilhada entre sexo, gênero e motricidade. Interamerican Journal of Psychology, 42(1), 69-79.

Carvalhal M., \& Vasconcelos-Raposo, J. (2007). Diferenças entre gêneros nas habilidades: Correr, saltar, lançar e pontapear. Motricidade, 3(3), 44-56.

Clark, J. E., \& Metcalfe, J. S. (2002). The mountain of motor development: A metaphor. In J. E. Clark, \& J. H. Humphrey (Eds.). Motor development: Research and reviews (Vol. II) (163-190). Reston, Va: NASPE.

Eikmann, S. H., Lira, P. I. C., Lima, M. C., Coutinho, S. B., Teixeira, M. L. P, \& Ashworth, A. (2007). Breast feeding and mental and motor development at 12 months in a low-income population in northeast Brazil. Paediatric and Perinatal Epidemiology, 21(2), 129-37. doi:10.1111/j.13653016.2007.00795.x

Fleuren, K. M. W., Smit, L. D., Stijnen, T., \& Hartman, A. (2007). New reference values for the Alberta Infant Motor Scale need to be established. Acta Paediatrica, 96(3), 424-427. doi: 10.1111/j.1651-2227.2007.00111.x.

Formiga, C. K., \& Linhares, M. B. (2011). Motor development curve from 0 to 12 months in infants born preterm. Acta Paediatrica, 100(3), 379-384. doi:10.1111/j.1651-2227.2010.02002.x

Gabbard, C., Caçola, P., \& Rodrigues, L. P. (2008). A new inventory for assessing affordances in the home environment for motor development (AHEMD-SR). Early Childhood Education Journal, 36(1), 5-9. doi:10.1007/s10643-008-0235-6

Gallahue, D. L., \& Ozmunm J. C. (2005). Compreendendo o desenvolvimento motor: Bebês, crianças, adolescentes e adultos. São Paulo: Phote.

Halpern, R., Giuliani, E. R. J., Victora, C. G., Barros, F. C., \& Horta, B. L. (2000). Fatores de risco para suspeita de atraso no desenvolvimento neuropsicomotor aos 12 meses de vida. Jornal de Pediatria, 76(6), 421-428. doi:10.4067/S0370-

\section{6}

Hamadani, J. D., Tofail, F., Hilaly, A., Huda, S. N., Engle, P., \& Grantham-McGregor, S. M. (2010). Use of family care indicators and their relationship with child development in Bangladesh. Journal of Health Popular Nutrition, 28(1), 23-33. doi:10.3329/jhpn.v28i1.4520

Haydari, A., Askari, P., \& Nezhad, M. Z. (2009). Relationship between affordances in the home enviroment and motor development in children age 18-42 months. Journal of Social Sciences, 5(4), 319-328. doi:10.3844/jssp.2009.319.328

Haywood, K. M., \& Getchell, N. (2010). Desenvolvimento motor ao longo da vida. Porto Alegre: ArtMed.

Lopes, V. B., Lima, C. D., \& Tudella, E. (2009). Motor acquisition rate in Brazilian infants. Infant and Child Development, 18(2), 122-132. doi:10.1002/ icd.595

Lung, F. W., Chiang, T. L., Lin, S. J., Feng, J. Y., Chen, P. F., \& Shu, B. C. (2011). Gender differences of children's developmental trajectory from 6 to 60 months in the Taiwan birth cohort pilot study. Research in Developmental Disabilities, 32(1), 100-106. doi:10.1016/j.ridd. 2010.09.004

Manacero, S., \& Nunes, M. L. (2008). Avaliação do desempenho motor de prematuros nos primeiros meses de vida na Escala Motora Infantil de Alberta (AIMS). Jornal de Pediatria, 84(1), 53-59. doi:10.1590/S0021-75572008000100009

Miranda, L. P., Resegue, R., \& Figueiras, A. C. M. (2003). A criança e o adolescente com problemas do desenvolvimento no ambulatório de pediatria. Jornal de Pediatria, 79(1), 133-42. doi:10.1590/S0021-75572003000700005

Nobre, F. S. S., Costa, C. L. A., Oliveira, D. L., Cabral, D. A., Nobre, G. C., \& Caçola, P. (2009). Análise das oportunidades para o desenvolvimento motor (affordances) em ambientes domésticos no Ceará - Brasil. Revista Brasileira de Crescimento e Desenvolvimento Humano, 19(1), 9-18.

Papalia, D. E., Olds, S. W., \& Feldman, R. T. (2010). Desenvolvimento Humano. Porto Alegre: Artmed.

Pierce, D., Munier, V., \& Myers, C. T. (2009). Informing early intervention through an occupa- 
tional science description of infant-toddler interactions with home space. The American Journal of Occupational Therapy, 63(3), 273-287. doi:10.5014/ajot.63.3.273

Pin, T., Eldridge, B., \& Galea, M. P. (2007). A review of the effects of sleep position, play position, and equipment use on motor development in infants. Developmental Medicine \& Child Neurology, 49(1), 858-867.

Piper, M. C., Pinnell, L. E., Darrah, J., Maguire, T., \& Byrne, P. J. (1992). Construction and validation of the Alberta Infant Motor Scale (AIMS). Canadian Journal Public Health, 83 (2), 46-50.

Pretti, L. C., Milan, J. C., \& Foschiani, M. A. (2010). Caracterização dos fatores ambientais e o controle cervical de lactentes nascidos pré-termo. Fisioterapia em Movimento, 23 (2), 239-250. doi:10.1590/S0103-51502010000200008

Saccani, R., \& Valentini, N. C. (2010). Análise do desenvolvimento motor de crianças de 0 a 18 meses de idade: Representatividade dos itens da Alberta Infant Motor Scale por faixa etária e postura. Revista Brasileira de Crescimento e Desenvolvimento Humano, 20(3), 753-764.

Santos, D. C., Campos, D., Gonçalves, V. M. G., Mello, B. B., Campos, T. M., \& Gagliardo, H. G. (2004). Influência do baixo peso ao nascer sobre o desempenho motor de lactentes a termo no primeiro semestre de vida. Revista Brasileira de Fisioterapia, 8(3), 261-266.

Schwengber, M. S. V. (2009). Meninas e meninos apresentam desempenho motor distinto? Por quê? Efedeports, 14(131). Retirado de http:// www.efdeportes.com/efd $131 /$ meninas-e-meninos-apresentam-desempenho-motor-distinto-por-que.htm

Thomas, J. R., \& French, K. E. (1985). Gender differences across age in motor performance: A meta-analysis. Psychological Bulletin, 98(2), 206-282. doi:10.1037/0033-2909.98.2.260
Thomas, J. R., \& Nelson, J. K. (2007). Métodos de pesquisa em atividade fisica. Porto Alegre: Artmed.

To, T., Guttmann, A., Dick, P. T., Rosenfield, J. D., Parkin, P. C., Cao, H.,...Harris, J. K. (2004). What factors are associated with poor developmental attainment in young Canadian children? Canadian Journal of Public Health, 95(4), 258-63.

Valentini, N. C. (2002). Percepções de competência e desenvolvimento motor de meninos e meninas: Um estudo transversal. Movimento, 8(2), 51-62.

Valentini, N. C., \& Saccani, R. (2011). Escala Motora Infantil de Alberta: Validação para uma população Gaúcha. Revista Paulista de Pediatria, 29(2), 231-8. doi:10.1590/S010305822011000200015

Wanderlind, F., Martins, G. D. F., Hansen, J., Macarini, S. M., \& Vieira, M. L. (2006). Diferenças de gênero no brincar de crianças pré-escolares e escolares na brinquedoteca. Revista Paidéia, 16(34), 263-273. doi:10.1590/S0103863X2006000200014

WHO Multicentre Growth Reference Study, \& Onis, M. (2006). Assessment of sex differences and heterogeneity in motor milestone attainment among populations in the WHO. Multicentre Growth Reference Study. Acta Pcediatrica, 450, 66-75. doi:10.1111/j.1651-2227.2006. tb02377.x

Zanini, P. Q., Hayashida, M., Hara, P. S., Lima, A. C., Castro, S. S., \& Bueno, C. F. (2002). Análise da aquisição do sentar, engatinhar e andar em um grupo de crianças pré-termo. Revista de Fisioterapia da Universidade de São Paulo, 9(2), 57-62.

(cc) EY-No Todo o conteúdo da revista Motricidade está licenciado sob a Creative Commons, exceto quando especificado em contrário e nos conteúdos retirados de outras fontes bibliográficas. 